\title{
INFLUENCE OF PROPIONALDEHYDE CONTAINING EXPERIMENTAL ADHESIVE SYSTEMS ON DENTIN BOND STRENGTH
}

\author{
Doaa Ahmed $^{*}$, Manal Soliman ${ }^{* *}$, Wafa El-Badrawy ${ }^{* * *}$ and Anuradha Prakki***
}

\begin{abstract}
This study was designed to investigate the influence of propionaldehyde priming on dentin microtensile bond strength ( $\mu \mathrm{TBS}$ ), and to evaluate the effect of storage on such bonds. Etched mid-coronal dentinal surfaces were treated with different priming procedures. Group PA: priming with experimental "primer A" composed of HEMA and ethanol; Group PPA: conditioning with propionaldehyde then priming with primer A; Group PB: priming with experimental "primer B" composed of HEMA and propionaldehyde. Specimens were then restored with composite resin and stored for $24 \mathrm{~h}, 6$ and 12 months prior to microtensile testing. Representative specimens were prepared and observed under SEM. Group PA showed significantly higher $\mu$ TBS at all testing times. Adhesive failure modes predominated in Group PB on immediate testing while no significant difference in failure modes distribution was noted at 6 and 12 months. Aging negatively affected $\mu$ TBS, with the least effect observed with the use of propionaldehyde/HEMA primer.
\end{abstract}

KEYWORDS: Propionaldehyde, Dentin, Microtensile Bond Strength, SEM

\section{INTRODUCTION}

Adhesive dentistry has been playing an integral role in many dental procedures. Nevertheless, the achievement of adequate dentinal bond strength and its durability remain of current interest and concern $^{1}$. Dentinal bond strengths are mainly affected by the stability of the hybrid layer ${ }^{2}$, which on the long term was shown to be affected by two degradation patterns, affecting the collagen network and the resin in the interfibrillar spaces ${ }^{1}$. Different strategies have been advocated and developed in order to prevent collagen and/or resin degradation, and therefore extend the longevity of dentinal bonds.

Stability of the demineralized collagen matrix and the improvement of the quality of demineralized dentin during resin infiltration have been demonstrated with the use of different aldehydes ${ }^{3,4}$. Dialdehydes were shown to act as collagen crosslinking agents ${ }^{5}$, fixing proteins irreversibly and increasing the modulus of elasticity of collagen

\footnotetext{
* Lecturer, Department of Restorative Dentistry, Faculty of Dentistry, Alexandria University, Alexandria, Egypt, ** Lecturer, Department of Restorative Dentistry, Faculty of Dentistry, Al Menia University, Al Menia, Egypt *** Associate Professor, Department of Clinical Sciences, Faculty of Dentistry, University of Toronto, Canada
} 
fibrils ${ }^{6}$. Their incorporation into adhesives ${ }^{7}$ or use as a primer solution ${ }^{8}$ was shown to positively impact the quality and longevity of the resin-dentin bonds. Nevertheless, aldehydes differ considerably in their ability to cross-link collagen both with respect to the number of cross links introduced as well as their stability ${ }^{9}$. The effect of monoaldehydes, such as propionaldehyde, on collagen cross linking remains controversial $^{4,9}$ and its ultimate effect on resin dentin bond strength has not yet been sufficiently investigated.

In regards to the resin stability, aldehydes were also shown to improve resin physical and mechanical properties. The ability of propionaldehyde to improve the properties of copolymers such as bisGMA/TEGDMA, UDMA/HEMA, and bis-GMA/ bis-GMA analogs have been demonstrated ${ }^{10,11}$. Incorporation of 24 to $32 \mathrm{~mol} \%$ propionaldehyde into dimethacrylates have shown significant increase on polymers degree of conversion ${ }^{12}$, thus improving mechanical properties such as flexural strength, elastic modulus and wear resistance, whereas of higher percentages of propionaldehyde reduced its effect, possibly due to the softening effect suppressing its cross-linking effect ${ }^{13}$.

In view of the above, it was of interest to study whether the suggested effects of propionaldehyde on demineralized dentinal collagen as well as on resins would ultimately affect the resin-dentin bond strength and its longevity. The aim of this study was to investigate the influence of propionaldehyde priming of demineralized dentin on dentinal bond strength and the effect of storage for 24h, 6 and 12 months. The following null hypotheses were tested: 1- the application of propionaldehyde to demineralized dentin prior to priming with HEMA/ ethanol or within an experimental primer does not affect the resin-dentin bond strength; 2 - aging does not affect the resin-dentin bond strength when dentin is treated with HEMA/ethanol primer or with propionaldehyde-containing priming protocol.

\section{MATERIALS AND METHODS}

\section{Experimental Design}

Microtensile testing was conducted to evaluate resin dentin bond strength as a response to the following two factors:

1- Treatment of demineralized dentin prior to bonding at three levels: Group PA - use of experimental primer without propionaldehyde (Primer A); Group PPA - dentin preconditioning with propionaldehyde followed by application of Primer A; and Group PB - use of experimental primer containing propionaldehyde (Primer B).

2- Aging of the bonded specimens at three levels: 24 hours (Immediate bonding); 6 months storage; and 12 months storage.

The association between the two factors ( 3 levels x 3 levels) resulted in 9 groups. Three extracted teeth were randomly assigned for each group.

\section{Materials}

The materials used are listed in Table 1.

\section{Experimental Primer Preparation}

Primer A: HEMA was combined with ethanol to establish a percentage ratio of 35/65 w/w \%, respectively ${ }^{8}$. Primer B: Propionaldehyde $97 \%$ was combined with HEMA to establish a ratio of $24 / 76$ mol\%. This was equivalent to $12.3 / 87.7 \mathrm{w} / \mathrm{w} \%$ in relevance to their molecular weight ${ }^{10}$.

\section{Specimen preparation}

Twenty-seven non-carious extracted human molars were used in this study. The teeth were selected from pooled and unidentified extracted teeth collected for research purposes at the department of Oral Surgery of the Faculty of Dentistry, University of Toronto. The teeth were sterilized by immersion in $2 \%$ chloramine $\mathrm{T}$ solution for one week then stored in non-ionized water until use. All teeth were used within 3 months of extraction. Flat, mid 
TABLE (1) Materials used in the study

\begin{tabular}{ccc}
\hline Materials & Composition & $\begin{array}{c}\text { Manufacturer } \\
\text { (Batch Number) }\end{array}$ \\
\hline Phosphoric Acid & 37\% Clear Phosphoric Acid Etchant & $\begin{array}{c}\text { 3M ESPE } \\
\text { St Paul, MN, USA (N274688) }\end{array}$ \\
\hline Propionaldehyde & Propionaldehyde 97\% & $\begin{array}{c}\text { Fluka Chemicals, Sigma-Aldrich } \\
\text { Chemie, GmbH, Steinheim, Germany } \\
\text { (08130MH) }\end{array}$ \\
\hline HEMA & 2-Hydroxyethylmetacrylate 99\% puriss.; & Fluka Chemicals, Sigma-Aldrich \\
& 0.001\% hydroquinone; monomethyl ether & Chemie, GmbH, Steinheim, Germany \\
as stabilizer & (09008HD)
\end{tabular}

coronal dentinal surfaces were prepared by grinding occlusal surfaces of teeth in a water-cooled grinding machine (Grinding and Polishing Machine, Struers Inc., 2750 Ballerup, Denmark) at $300 \mathrm{rpm}$. A surface smear-layer was created by $10 \mathrm{~s} / 300 \mathrm{rpm}$ grinding with 600 grit silicon-carbide abrasive papers. Prior to bonding, exposed flat dentinal surfaces of all specimens were etched (37\% phosphoric acid for 15 seconds), rinsed with water for 10 seconds then wiped dry with absorbent paper leaving a moist dentinal surface.

\section{Definition of Groups and Bonding procedures}

The prepared teeth were randomly divided into 3 groups ( $n=9)$ according to the different dentin conditioning and priming procedures described in Table 2. Once primed, all specimens of the three groups were coated with 2 layers of adhesive (Scotchbond Adper Multi-Purpose Plus Dental Adhesive, 3M ESPE) and light-cured for 10 seconds using an LED light cure unit (Demi, SDS Kerr Corp., Middleton, WI, USA). The intensity of light delivered was $984 \mathrm{~mW} / \mathrm{cm}^{2}$ as premeasured using a curing light testing device (CheckMARC, BlueLight Analytics, Halifax, NS, CA). For each tooth, a $3 \mathrm{~mm}$ composite core buildup was made in two increments, each $1.5 \mathrm{~mm}$ thick, using Filtek Z250 universal restorative resin, shade A2 (3M, ESPE), and light cured for 20 seconds per increment. Restored teeth were then placed in deionized water and incubated at $37^{\circ} \mathrm{C}$ until testing (24 hours, 6 months, and 12 months). Stored specimens were periodically rinsed and placed in fresh deionized water until testing time.

\section{Microtensile Bond Strength Testing (MPa)}

Following the assigned storage period, specimens were serially sectioned into $1 \mathrm{~mm}$-thick slabs using a low speed diamond saw (Isomet, Buehler Ltd, Lake Bluff, IL, USA). Each slab was 
TABLE (2) Different conditioning and priming protocols used in the study

\begin{tabular}{|c|c|c|}
\hline Groups & Pre-conditioning & Priming \\
\hline PA & - & Apply two coats of primer $A^{*}$, slight air dry \\
\hline PPA & $\begin{array}{l}\text { Apply two coats of propionaldehyde**, } \\
\text { wipe dry with absorbent paper }\end{array}$ & Apply two coats of primer $A^{*}$, slight air dry \\
\hline $\mathrm{PB}$ & _- & Apply two coats of primer $\mathrm{B}^{* * *}$, slight air dry \\
\hline \multicolumn{3}{|c|}{ *Primer A: HEMA/ethanol 35/65 w/w \% } \\
\hline ***Propiona & $97 \%$ & \\
\hline
\end{tabular}

then rotated 90 degree and sectioned again to obtain $6 \mathrm{~mm}$ long resin-dentin beams with a cross-sectional area of $1 \mathrm{~mm}^{2}(+/-0.1 \mathrm{~mm})$. The bonded surface area was calculated before each test by measuring the narrowest portion of the interface to the nearest $0.01 \mathrm{~mm}$ using a digital caliper (Mastercraft electronic caliper, Canadian Tire Corporation, Ltd, ON, Canada). Beams were fixed to the microtensile jig using cyanoacrylate glue (Zapit, Dental Ventures of America, Corona, CA, USA), and were subjected to microtensile bond testing using microtensile testing machine (Bisco Inc., Schaumburg, IL, USA) at a crosshead speed of $0.5 \mathrm{~mm} / \mathrm{min}$.

\section{Fracture Mode Analysis}

All specimens subjected to microtensile bond testing were observed under optical microscope (Olympus corporation, model CKX41, Tokyo, Japan) at $40 \times$ magnification to identify the mode of fracture. Fracture modes were classified into cohesive failures in dentin (Coh.D), cohesive failure in adhesive layer (Coh.AD), cohesive failures in composite (Coh. C), adhesive failures between dentin and adhesive layer (Adh.AD), adhesive failures between adhesive layer and composite (Adh.AC) and combination failures where hybrid fracture occurred at more than 1 location (Comb.). The percentage of each fracture mode was then calculated for each group.

\section{Scanning Electron Microscope (SEM) Protocol}

Representative samples of each group were additionally prepared for SEM analysis of the hybrid layer. Two resin-dentin slices were obtained from the central portion of each restored tooth. Specimens were fixed with Karnovsky's fixative (16\% paraformaldehyde, 1M sodium hydroxide, $50 \%$ glutaraldehyde in $0.2 \mathrm{M}$ cacodylate buffer, $\mathrm{pH}$ 7.4) before and after sectioning for 24 hours each. For each specimen, the surface to be examined was finished by the sequence of 600, 800, 1000 and 1200 grit silicon-carbide abrasive papers under running water for 20 seconds each, and polished with a sequence of 3.0 and $0.5 \mu \mathrm{m}$ aluminum oxide slurries (for 15 seconds each). All slices were subjected to 5 minutes ultrasonic bath after each polishing cycle. The polished surfaces were etched with $37 \%$ phosphoric acid for 10 seconds and then immersed for 15 minutes in $6 \%$ NaOCL solution to allow deproteinization and to reveal the resin interfusion zone. Specimens were then subjected to a graded alcohol dehydration protocol ${ }^{1}$. Once dehydrated, the specimens were placed in a critical point dryer, mounted on aluminum stubs, coated with 6 $\mathrm{nm}$ platinum layer (Polaron SC515, SEM coating System, Fisons, Bell Lane Vkfield, VG Micorotech, UK) and examined with SEM (Hitachi S25000, Lld 
Mito City, Japan). Photomicrographs of the resin dentin interface representing the different groups were obtained.

\section{Statistical Analysis}

The microtensile bond strength data were analyzed by one-way ANOVA followed by Tukey's post-hoc test. Comparison of the distribution of failure modes within same testing group and/or same testing time was done using chi square test. The statistical significance was preset at $5 \%$.

\section{RESULTS}

The microtensile bond strength mean values are summarized in Table 3. Group PA showed significantly higher bonds strengths compared to Group PPA and Group PB $(p<0.001)$, at all testing periods. There was no significant difference between the bond strength values obtained from Groups PPA and PB at immediate testing, 6 months or 12 months $(p=0.161)$. In all groups (PA, PPA and PB), the 6 months and 12 months aged samples showed significantly lower bond strength values when compared to their respective immediate testing results $(p<0.001)$. Nevertheless, the percentage reduction in bond strength varied among groups, with group PB showing least percentage decrease in bond strength with storage. There was no significant difference in bond strength between the 6 and the 12 months aged samples for any of the evaluated groups $(p=0.681)$.

The percentage fracture mode distribution among different groups is shown in Table 4. At immediate testing, there was a significant fracture mode difference among evaluated groups $(p=0.002)$ with Group PB being significantly different from Groups PA and PPA. It was observed that in Group PA, cohesive failures ("Coh.D" and "Coh.C") predominated, while in Group PB, adhesive failures were predominant with $55.6 \%$ of the specimens showing "Adh.AD" mode. There was no significant difference in failure modes among the different groups at 6 months $(p=0.09)$ or 12 months $(p=0.83)$ testing periods, with the "Adh.AD" being most common for all groups. Across the different testing times, there was a statistical difference between the failure modes in group PA $(p=0.05)$ and group PPA ( $p=0.002)$. In group PA at immediate testing, cohesive failure modes were most common, but at 6 and 12 months, "Adh.AD" became prevalent. The same was observed in group PPA at 6 and 12 months testing. As for group PB, no statistically significant difference $(p=0.65)$ was observed between the distribution of the different failure modes across the different testing times, with the "Adh.AD" being most common.

TABLE (3) Microtensile bond strength mean values (MPa) and standard deviations (S.D.)

\begin{tabular}{|c|c|c|}
\hline Groups & Period of Evaluation & $\begin{array}{c}\text { Bond Strength } \\
(\text { S.D })\end{array}$ \\
\hline \multirow{3}{*}{ Group PA } & Immediate & $57.8(17.0)^{\mathrm{a}}$ \\
\cline { 2 - 3 } & 6 months & $40.4(14.5)^{\mathrm{b}}$ \\
\cline { 2 - 3 } Group & 12 months & $40.4(16.1)^{\mathrm{b}}$ \\
\cline { 2 - 3 } PPA & Immediate & $46.4(18.5)^{\mathrm{c}}$ \\
\cline { 2 - 3 } & 6 months & $26.7(9.0)^{\mathrm{d}}$ \\
\hline \multirow{3}{*}{ Group PB } & 12 months & $25.6 \pm 13.3^{\mathrm{d}}$ \\
\cline { 2 - 3 } & Immediate & $38.4(9.5)^{\mathrm{c}}$ \\
\cline { 2 - 3 } & 12 months & $22.3(9.2)^{\mathrm{d}}$ \\
\hline
\end{tabular}

Different superscript letters denote statistically significant differences $(\alpha=0.05)$ 
TABLE (4) Percentage of fracture mode in the different groups

\begin{tabular}{|c|c|c|c|c|c|c|}
\hline \multirow{2}{*}{$\begin{array}{c}\text { Groups } \\
\text { Evaluation Period }\end{array}$} & \multicolumn{3}{|c|}{ Cohesive Fracture } & \multicolumn{2}{|c|}{ Adhesive Fracture } & \multirow{2}{*}{$\begin{array}{c}\text { Combination } \\
\text { (Comb. })\end{array}$} \\
\hline & (Coh. D) & (Coh. A) & (Coh.C) & (Adh. AD) & (Adh. AC) & \\
\hline Group PA Immediate $^{a}$ & 28.6 & 14.3 & 28.6 & 14.3 & 0.0 & 14.3 \\
\hline Group PPA Immediate & 25.0 & 10.7 & 10.7 & 21.4 & 14.3 & 17.9 \\
\hline Group PB Immediate $^{b}$ & 7.4 & 22.2 & 0.0 & 55.6 & 7.4 & 7.4 \\
\hline $\begin{array}{l}\text { Group } \mathrm{PA}^{\mathrm{c}} \\
6 \text { months }\end{array}$ & 20.7 & 17.2 & 6.9 & 41.4 & 3.4 & 10.3 \\
\hline $\begin{array}{c}\text { Group PPA }{ }^{\mathrm{c}} \\
6 \text { months }\end{array}$ & 3.3 & 6.7 & 0.0 & 73.3 & 0.0 & 16.7 \\
\hline $\begin{array}{l}\text { Group } \mathrm{PB}^{\mathrm{c}} \\
6 \text { months }\end{array}$ & 3.4 & 17.2 & 0.0 & 58.6 & 3.4 & 17.2 \\
\hline $\begin{array}{l}\text { Group } \text { PA }^{\mathrm{d}} \\
12 \text { months }\end{array}$ & 6.9 & 20.7 & 6.9 & 51.7 & 0.0 & 13.8 \\
\hline $\begin{array}{c}\text { Group PPA }^{\mathrm{d}} \\
12 \text { months }\end{array}$ & 11.1 & 11.1 & 3.7 & 66.7 & 0.0 & 7.4 \\
\hline $\begin{array}{l}\text { Group } \mathrm{PB}^{\mathrm{d}} \\
12 \text { months }\end{array}$ & 3.4 & 13.8 & 6.9 & 65.5 & 3.4 & 6.9 \\
\hline
\end{tabular}

Different superscript letters denote statistically significant differences $(\alpha=0.05)$ between different groups, within each testing time.

\section{DISCUSSION}

As stated, three experimental priming protocols were used in this study. The SEM images (Figure 1a-1f) confirmed that all systems were capable of producing hybrid layers with resin penetration as well as tag formation. The bond strength data indicated that the use of propionaldehyde in dentin priming protocols rendered adequate bond strength ranging from 38.4 MPa to 46.4 MPa (Table 3). Nevertheless, the use of HEMA/ethanol primer (group PA) still showed significantly higher bond strength (40.4 MPa to 57.8 MPa). The null hypothesis that incorporation of propionaldehyde in the preconditioning or priming of demineralized dentin does not affect the resin-dentin bond strength was therefore rejected. These results are in line with those obtained by Asmussen et al.$^{4}$ who emphasized on the use of aldehydes in combination with HEMA as dentin adhesion promotors. They have suggested that the aldehyde primarily reacts with dentinal collagen forming an $\mathrm{N}$-(hydroxyalkyl) intermediate. HEMA then reacts with this collagen-aldehyde intermediate allowing subsequently applied resins to copolymerize with the collagen-linked methacrylate groups. Of the various aliphatic aldehydes tested, propionaldehyde, in combination with HEMA, exhibited the highest tensile bond strength compared to other aromatic and heteroaromatic aldehydes tested. In accordance, Munksgaard ${ }^{14}$ also observed a $\mathrm{pH}$ dependent precipitation of glycine aminoacid with the addition of $10 \%$ propionaldehyde in HEMA suggesting the formation of a cross-linked polymer. 


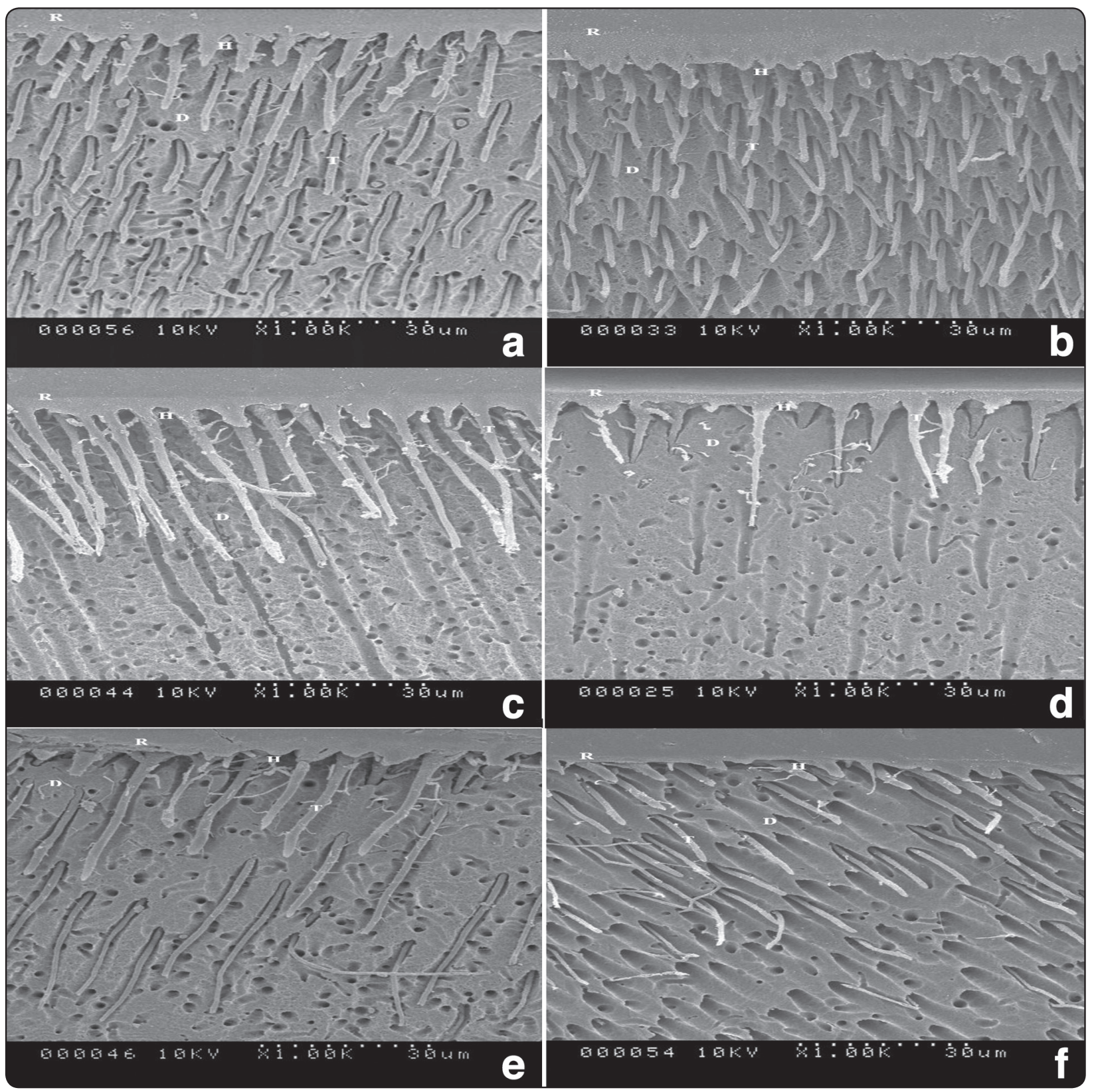

Fig. (1) Representative micrographs of the hybrid layers produced: (a) Group PA -immediate; (b) Group PA- 1 year; (c) Group PPA - immediate; (d) Group PPA - 1 year; (e) Group PB - immediate; (f) Group PB - 1 year. R: resin composite, H: hybrid layer, D: dentin, T: resin tags.

Nevertheless, there appears to be conflicting evidence in regards to the reliable collagen crosslinking ability of propionaldehyde compared to more intensively investigated aldehydes such as formaldehyde and glutaraldehyde. Cox et al. ${ }^{15}$ supported the view that propionaldehyde and acetaldehyde react extensively with collagen and demonstrated by amino acid analyses that propionaldehyde gave very insoluble and stable products, comparable to the product obtained with glutaraldehyde. In contrast, Bowes et al. ${ }^{9}$ found that propionaldehyde was relatively unreactive towards amino groups compared to formaldehyde even though the bonds formed by the latter appeared to be 
relatively easily broken. It has also been reported that the ability of propionaldehyde to form DNA-protein crosslinks were of several orders of magnitude, being however less reactive than formaldehyde and unsaturated aldehydes ${ }^{16}$. This weaker effect could be attributed to the fact that propionaldehyde is a saturated primary aldehyde having one functional group, with which the most relevant reaction is nucleophilic addition ${ }^{17}$. This reaction is usually reversible, in contrast to glutaraldehyde capable of alpha-beta unsaturated aldehyde aldol condensation reaction, which may undergo irreversible additions to the double bond as well as to the aldehyde ${ }^{14,15}$. In this context, the reported benefits of glutaraldehyde on cross-linking and fixing demineralized dentin collagen ${ }^{18}$, thus positively impacting resin-dentin bond strength ${ }^{8}$, could not be duplicated in this study with the use of propionaldehyde.

On the other hand, the ability of propionaldehyde to influence physical and mechanical properties of resins has been demonstrated ${ }^{10-13}$. Peutzfeldt et al..$^{10}$ found an improvement in diametral tensile strength, flexural strength, and modulus of elasticity of resins with the addition of propionaldehyde, a potential cross-linking agent, to light-curable bis-GMA/ TEGDMA and UEDMA/HEMA based materials. They later investigated the effect of propionaldehyde on chemically-curable bis-GMA/TEGDMA monomers mixtures, putting light on the significant decrease in quantity of remaining double bonds to the evaluated systems ${ }^{19}$. Propionaldehyde would act as a chain transfer agent, acting as a free radical and, because of its small size, would react with double bonds that otherwise would not have reacted ${ }^{11,12,19,20}$. Such positive effect of propionaldehyde additives would be expected to subsequently reflect on the resistance to degradation of the resin at the hybrid layer and thus improve durability of the bond. Nevertheless, in the current study, propionaldehyde was combined with HEMA in a primer solution (Primer PB) and subjected to a moist dentinal environment. Whether its incorporation in such manner would ultimately affect the properties of the adhesive resin within the hybrid layer remains to better investigated.

In respect to aging, all groups in the current study showed a significant decrease in bond in the first 6 months, with relative stability from 6 to 12 months, which prompts the rejection of the second proposed null hypothesis. Nevertheless, even though bond strength in all groups tested was significantly lower after 6 months storage compared to baseline, the effect on storage was more marked in Group PA compared to Group PB (30.1\% and $19.7 \%$ decrease in bond strength respectively). This may suggest some reaction between propionaldehyde and HEMA in an unpolymerized state in group PB. On the other hand, the use of propionaldehyde as dentin conditioning prior to use of primer A (group PPA) showed the greatest drop in bond strength at 6 months compared to baseline (42.4\%). This may be attributed to the reported ability of propionaldehyde to increase water sorption of resin ${ }^{21}$, consequently making the resin more susceptible to hydrolytic degradation ${ }^{1}$. Another plausible explanation refers to the assumption that propionaldehyde reacts reversibly with collagen ${ }^{16}$. Propionaldehyde could have primarily reacted with collagen and was not satisfactorily available to react with and increase HEMA cross-linking when subsequently applied within primer $A$. The reversible nature of this reaction with collagen could have revealed its effect with aging, resulting in the observed drop in group PPA bond strength.

The bond failures observed in the current study correlated well to the bond strength values obtained in the different groups. At baseline, Group PA showed higher cohesive failures, either in dentin or in composite, which suggested a strong bond at the interface reflected in the higher bond strength obtained in that group (57.8 MPa). Percentages failure mode in group PPA with bond strength of 46.4 MPa, were not significantly different than in Group PA, even though more adhesive failures were registered. As for the group PB, failure modes were significantly different from groups PA and PPA, with adhesive failures predominating. This as 
well reflects the significantly lower bond strength (38.4 MPa) obtained in this group. It was also noted that aging had an effect on the failure mode distribution, with adhesive failure modes predominating in all groups, which may explain the lower bond strength obtained at 6 and 12 months.

\section{CONCLUSION}

Under the conditions of the current study, it could be concluded that the use of propionaldehyde as dentin conditioning or in an experimental primer has reduced resin-dentin bond strengths compared to the use of HEMA/ethanol experimental primer. Aging significantly decreased the dentinal bond strength of all systems evaluated, with the least effect observed with the use of propionaldehyde/ HEMA experimental primer.

\section{ACKNOWLEDGMENTS}

This study was supported by Connaught New Researcher Award to AP. The authors would like to extend their thanks and appreciation to 3M-ESPE for supplying some of the commercial materials.

\section{REFERENCES}

1. Hashimoto M, Ohno H, Sano H, Kaga M, Oguchi H. In vitro degradation of resin-dentin bonds analyzed by microtensile bond test, scanning and transmission electron microscopy. Biomater 2003; 24: 3795-3803.

2. Nakabayashi N, Kojimi K, Masuhar E. The promotion of adhesion by the infiltration of monomers into tooth substrates. J Biomed Mater Res 1982; 16: 265-273.

3. Kiernan JA. Formaldehyde, formalin, paraformaldehyde and glutaraldehyde: What they are and what they do. Microscop Today 2000; 00-1: 8-12.

4. Asmussen E and Munksgaard EC. Bonding of restorative resins to dentine promoted by aqueous mixtures of aldehydes and active monomers. Int Dent J 1985; 35: 160-165.

5. Al-Ammar A, Drummond JL, Bedran-Russo AK. The use of collagen cross-linking agents to enhance dentin bond strength. J Biomed Mater Res B Appl Biomater 2009; 91: 419-24.

6. Bedran-Russo AK, Pereira PN, Duarte WR, Drummond JL, Yamauchi M. Application of crosslinkers to dentin collagen enhances the ultimate tensile strength. J Biomed Mater Res B: Appl Biomater 2007; 80: 268-272.
7. Xu C, Wang Y. Collagen cross linking increases its biodegradation resistance in wet dentin bonding. J Adhes Dent 2012; 14:11-18.

8. Cilli R, Prakki A, de Araújo PA, Pereira JC. Influence of glutaraldehyde priming on bond strength of an experimental adhesive system applied to wet and dry dentine. J Dent 2009; 37:212-218.

9. Bowes $\mathrm{J} \mathrm{H}$, Cater $\mathrm{CW}$. The interaction of aldehydes with collagen. Biochim Biophys Acta 1968; 168: 341-352.

10. Peutzfeldt A, Asmussen E. Influence of aldehydes on selected mechanical properties of resin composites. J Dent Res 1992; 71: 1522-1524.

11. Prakki A, Pereira PN, Kalachandra S. Effect of propionaldehyde or 2,3-butanedione additives on the mechanical properties of Bis-GMA analog-based composites. Dent Mater 2009; 25: 26-32.

12. Prakki A, Tallury P, Mondelli RF, Kalachandra S. Influence of additives on the properties of Bis-GMA/Bis-GMA analog comonomers and corresponding copolymers. Dent Mater 2007; 23: 1199-1204.

13. Peutzfeldt A, Asmussen E. In vitro wear, hardness, and conversion of diacetyl-containing and propanal-containing resin materials. Dent Mater 1996; 12: 103-108.

14. Munksgaard EC. Amine-induced polymerization of aqueous HEMA/aldehyde during action as a dentin bonding agent. J Dent Res 1990; 69:1236-1239.

15. Cox RW, Grant RA, Kent CM. An electron-microscope study of the reaction of collagen with some monoaldehydes and bifunctional aldehydes. J Cell Sci 1973; 12: 933-949.

16. Kuykendall JR, Bogdanffy MS. Efficiency of DNA-histone crosslinking induced by saturated and unsaturated aldehydes in vitro. Mutat Res 1992; 283:131-136.

17. Guth DJ. Dose-response assessment of non-cancer effects of propionaldehyde based on comparative toxicity with other short-chain aldehydes. Hum Ecol Risk Asses 1996; 2: $580-590$

18. Carvalho RM, Mendonça JS, Santiago SL, Silveira RR, Garcia FC, Tay FR, Pashley DH. Effects of HEMA/solvent combinations on bond strength to dentin. J Dent Res 2003; 82: 597-601.

19. Peutzfeldt A, Asmussen E. Effect of propanal and diacetyl on quantity of remaining double bonds of chemically cured BisGMA/TEGDMA resins. Eur J Oral Sci 1996; 104: 309-312.

20. Peutzfeldt A. Retention of propanal and diacetyl in experimental resins. Acta Odontol Scand 1997; 55: 94-100.

21. Prakki A, Cilli R, Vieira IM, Dudumas K, Pereira JC. Water sorption of $\mathrm{CH} 3$ - and CF3-Bis-GMA based resins with additives. J Appl Oral Sci 2012; 20: 472-477. 